\title{
The CCFM Monte Carlo generator CASCADE Version 2.2.03
}

\author{
H. Jung ${ }^{1,2, a}$, S. Baranov ${ }^{3}$, M. Deak ${ }^{4}$, A. Grebenyuk ${ }^{1}$, F. Hautmann ${ }^{5}$, M. Hentschinski ${ }^{1}$, A. Knutsson ${ }^{1}$, M. Krämer ${ }^{1}$, \\ K. Kutak ${ }^{2}$, A. Lipatov ${ }^{6}$, N. Zotov 6 \\ ${ }^{1}$ DESY, Hamburg, Germany \\ ${ }^{2}$ University of Antwerp, Antwerp, Belgium \\ ${ }^{3}$ Lebedev Physics Institute, Moscow, Russia \\ ${ }^{4}$ Instituto de Física Teórica UAM/CSIC, University of Madrid, Madrid, Spain \\ ${ }^{5}$ University of Oxford, Oxford, UK \\ ${ }^{6}$ SINP, Moscow State University, Moscow, Russia
}

Received: 19 August 2010 / Revised: 31 October 2010 / Published online: 20 November 2010

(C) The Author(s) 2010. This article is published with open access at Springerlink.com

\begin{abstract}
CASCADE is a full hadron level Monte Carlo event generator for $e p, \gamma p$ and $p \bar{p}$ and $p p$ processes, which uses the CCFM evolution equation for the initial state cascade in a backward evolution approach supplemented with off-shell matrix elements for the hard scattering. A detailed program description is given, with emphasis on parameters the user wants to change and common block variables which completely specify the generated events.
\end{abstract}

\section{Program summary}

Title of Program: CASCADE 2.2.03

Computer for which the program is designed and others on which it is operable: any with standard Fortran 77 (g77 or gfortran), tested on SGI, HP-UX, SUN, PC, MAC

Programming Language used: FORTRAN 77

High-speed storage required: No

Separate documentation available: No

Keywords: QCD, small $x, k_{t}$-factorization, CCFM, parton showers, leptoproduction, photoproduction, $p p$ - and $p \bar{p}$ scattering, heavy quark production, unintegrated PDFs.

Nature of physical problem: High-energy collisions of particles at moderate values of the fractional momentum $x$ are well described by resummation of leading logarithms of transverse momenta $\left(\alpha_{\mathrm{S}} \ln Q^{2}\right)^{n}$, generally referred to as DGLAP physics. At small $x$ leading-logs of longitudinal momenta, $\left(\alpha_{\mathrm{s}} \ln x\right)^{n}$, are expected to become equally if not more important (BFKL). An appropriate description valid

a e-mail: hannes.jung@desy.de for both small and moderate $x$ is given by the CCFM evolution equation, resulting in an unintegrated gluon density $\mathcal{A}\left(x, k_{t}, \bar{q}\right)$, which is also a function of the evolution scale $\bar{q}$.

Method of solution: Since measurements involve complex cuts and multi-particle final states, the ideal tool for any theoretical description of the data is a Monte Carlo event generator which embodies small- $x$ resummation, in analogy to event generators which use DGLAP resummation. The CCFM evolution equation forms a bridge between the DGLAP and BFKL resummation and can be applied to generate the initial state branching processes. The CCFM equation can be formulated in a way suitable for carrying out a backward evolution, which is an essential requirement to efficiently generate unweighted Monte Carlo events.

Restrictions on the complexity of the problem: The following hard subprocesses can be simulated:

$\gamma^{*} g^{*} \rightarrow q \bar{q}(Q \bar{Q})$,

$\gamma g^{*} \rightarrow J / \psi g$

$g^{*} g^{*} \rightarrow q \bar{q}(Q \bar{Q})$,

$g^{*} g^{*} \rightarrow J / \psi g$

$g^{*} g^{*} \rightarrow \chi$,

$g^{*} g \rightarrow g g$,

$g^{*} q \rightarrow g q$,

$g^{*} g^{*} \rightarrow h^{0}$

$g^{*} q \rightarrow Z(W) q$,

$g^{*} g^{*} \rightarrow Z Q \bar{Q}, g^{*} g^{*} \rightarrow Z q \bar{q}$,

$g^{*} g^{*} \rightarrow W q_{i} q_{j}$.

The present version is applicable for HERA, TEVATRON and LHC processes.

Other Program used: PYTHIA (version > 6.4) for hadronization, BASES/SPRING 5.1 for integration (supplied with the program package). 


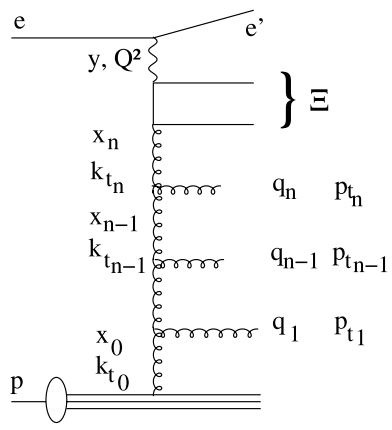

(a)

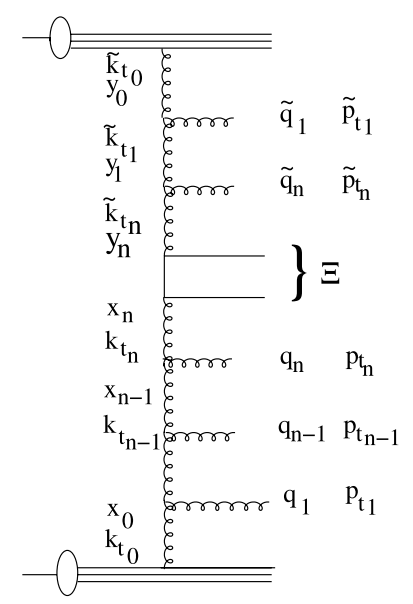

(b)
Fig. 1 Kinematic variables for multi-gluon emission in leptoproduction (a) and hadroproduction (b). The $t$-channel gluon four-vectors are given by $k_{n}$ and the gluons emitted in the initial state cascade have four-vectors $p_{n}$. The maximum angle (a function of the rapidity) for any emission is obtained from the quark box, as indicated with $\Xi$

\section{Download of the program: http://www.desy.de/ jung/} cascade

Unusual features of the program: None

\section{The CCFM evolution equation}

The formulation of the CCFM [1-4] parton evolution for the implementation into a full hadron level Monte Carlo program is described in detail in [5, 6]. Here only the main results are summarized and discussed. The pattern of QCD initial state radiation in a small- $x$ event in $e p$ and $p \bar{p}(p p)$ collisions is illustrated in Fig. 1 together with labels for the kinematics. According to the CCFM evolution equation, the emission of partons during the initial cascade is only allowed in an angular ordered region of phase space. In terms of Sudakov variables $\Upsilon$ and $\Xi$ the quark pair momentum is written as:

$p_{q}+p_{\bar{q}}=\Upsilon\left(p^{(1)}+\Xi p^{(2)}\right)+Q_{t}$,

where $p^{(1)}$ and $p^{(2)}$ are the four-vectors of incoming particles (electron-proton, proton-antiproton or proton-proton), respectively and $Q_{t}$ is the transverse momentum of the quark pair in the center of mass frame of $p^{(1)}$ and $p^{(2)}(\mathrm{cms})$. The variable $\Xi$ is related to the rapidity $Y$ in the center of mass (CMS) frame via

$Y=\frac{1}{2} \log \left(\frac{E+p_{z}}{E-p_{z}}\right)=\frac{1}{2} \log \left(\frac{1}{\Xi}\right)$.
Using $E=E_{q}+E_{\bar{q}}$ and $p_{z}=p_{q z}+p_{\bar{q} z}$ gives $E+p_{z}=$ $\Upsilon \sqrt{s}, E-p_{z}=\Upsilon \Xi \sqrt{s}$ with $E=\sqrt{s} / 2$ and $s=\left(p^{(1)}+\right.$ $\left.p^{(2)}\right)^{2}$ being the squared center of mass energy. Therefore $\Xi$ can be used to define the maximum allowed angle in the evolution. The momenta $p_{i}$ of the gluons emitted during the initial state cascade are given by (here treated massless):

$p_{i}=v_{i}\left(p^{(1)}+\xi_{i} p^{(2)}\right)+p_{t i}, \quad \xi_{i}=\frac{p_{t i}^{2}}{s v_{i}^{2}}$,

with $v_{i}=\left(1-z_{i}\right) x_{i-1}$ and $x_{i}=z_{i} x_{i-1}$. The variables $x_{i}$ and $v_{i}$ are the momentum fractions of the exchanged and emitted gluons, while $z_{i}$ is the momentum fraction in the branching $(i-1) \rightarrow i$ and $p_{t i}$ is the transverse momentum of the emitted gluon $i$. Again the rapidities $y_{i}$ are given by $y_{i}=-0.5 \log \xi_{i}$ in the CMS frame.

The angular ordered region is then specified by (Fig. 1a and the lower part of the cascade in Fig. 1b, for the upper part the variables have to be changed accordingly):

$\xi_{0}<\xi_{1}<\cdots<\xi_{n}<\Xi$

which becomes:

$z_{i-1} q_{i-1}<q_{i}$,

where the rescaled transverse momentum $q_{i}$ of the emitted gluon is defined by:

$q_{i}=x_{i-1} \sqrt{s \xi_{i}}=\frac{p_{t i}}{1-z_{i}}$.

The scale $\bar{q}$ (related to the maximum angle) can be written as:

$\bar{q}^{2}=\Upsilon^{2} \Xi_{s}=\hat{s}+Q_{t}^{2}$,

with $\hat{s}=\left(p_{q}+p_{\bar{q}}\right)^{2}$ and the relation of $\bar{q}$ to a particular choice of the factorization scale $\mu_{f}$ in the collinear approach becomes obvious.

The CCFM evolution equation can be written in a differential form [4], which is best suited for a backward evolution approach adopted in the Monte Carlo generator CASCADE $[5,6]$ :

$$
\begin{aligned}
& \bar{q}^{2} \frac{d}{d \bar{q}^{2}} \frac{x \mathcal{A}\left(x, k_{t}, \bar{q}\right)}{\Delta_{s}\left(\bar{q}, Q_{0}\right)} \\
& \quad=\int d z \frac{d \phi}{2 \pi} \frac{\tilde{P}\left(z, \bar{q} / z, k_{t}\right)}{\Delta_{s}\left(\bar{q}, Q_{0}\right)} x^{\prime} \mathcal{A}\left(x^{\prime}, k_{t}^{\prime}, \bar{q} / z\right),
\end{aligned}
$$

where $\mathcal{A}\left(x, k_{t}, \bar{q}\right)$ is the unintegrated gluon density, depending on $x, k_{t}$ and the evolution variable $\bar{q}$. The splitting variable is $z=x / x^{\prime}$ and $\boldsymbol{k}_{t}^{\prime}=(1-z) / z \boldsymbol{q}+\boldsymbol{k}_{t}$, where the vector $\boldsymbol{q}$ is at an azimuthal angle $\phi$. The Sudakov form factor $\Delta_{S}$ is 
given by:

$\Delta_{s}\left(\bar{q}, Q_{0}\right)=\exp \left(-\int_{Q_{0}^{2}}^{\bar{q}^{2}} \frac{d q^{2}}{q^{2}} \int_{0}^{1-Q_{0} / q} d z \frac{\bar{\alpha}_{s}\left(q^{2}(1-z)^{2}\right)}{1-z}\right)$,

with $\bar{\alpha}_{s}=\frac{C_{A} \alpha_{s}}{\pi}=\frac{3 \alpha_{s}}{\pi}$. For inclusive quantities at leadinglogarithmic order the Sudakov form factor cancels against the $1 /(1-z)$ collinear singularity of the splitting function.

The original splitting function $\tilde{P}_{g}\left(z_{i}, q_{i}, k_{t i}\right)$ for branching $i$ is given by (neglecting finite terms as they are not obtained in CCFM at the leading infrared accuracy (cf p. 72 in [3]):

$\tilde{P}_{g}\left(z_{i}, q_{i}, k_{t i}\right)=\frac{\bar{\alpha}_{s}\left(q_{i}^{2}\left(1-z_{i}\right)^{2}\right)}{1-z_{i}}+\frac{\bar{\alpha}_{s}\left(k_{t i}^{2}\right)}{z_{i}} \Delta_{n s}\left(z_{i}, q_{i}^{2}, k_{t i}^{2}\right)$,

where the non-Sudakov form factor $\Delta_{n s}$ is defined as:

$\log \Delta_{n s}=-\bar{\alpha}_{s}\left(k_{t i}^{2}\right) \int_{0}^{1} \frac{d z^{\prime}}{z^{\prime}} \int \frac{d q^{2}}{q^{2}} \Theta\left(k_{t i}-q\right) \Theta\left(q-z^{\prime} q_{t i}\right)$.

The implementation of the full splitting function including non singular terms can lead to inconsistencies. Replacing naively only $\frac{1}{1-z} \rightarrow \frac{1}{1-z}-2+z(1-z)$ in the CCFM splitting function can lead to negative branching probabilities.

In [7] it was suggested to use:

$$
\begin{aligned}
P(z, q, k)= & \bar{\alpha}_{s}\left(k_{t}^{2}\right)\left(\frac{(1-z)}{z}+(1-B) z(1-z)\right) \\
& \times \Delta_{n s}(z, q, k) \\
& +\bar{\alpha}_{s}\left((1-z)^{2} q^{2}\right)\left(\frac{z}{1-z}+B z(1-z)\right),
\end{aligned}
$$

where $B$ is a parameter to be chosen arbitrarily between 0 and 1 , we take $B=0.5$. As a consequence of the replacement, the Sudakov form factor will change, but also the nonSudakov form factor needs to be replaced by:

$$
\begin{aligned}
\log \Delta_{n s}= & -\bar{\alpha}_{s}\left(k_{t}^{2}\right) \int_{0}^{1} d z^{\prime}\left(\frac{1-z}{z^{\prime}}+(1-B) z(1-z)\right) \\
& \times \int \frac{d q^{\prime 2}}{q^{\prime 2}} \Theta\left(k-q^{\prime}\right) \Theta\left(q^{\prime}-z^{\prime} q\right) .
\end{aligned}
$$

\section{Backward evolution: CCFM and CASCADE}

The idea of a backward evolution $[8,9]$ is to first generate the hard scattering process with the initial parton momenta distributed according to the parton distribution functions. This involves in general only a fixed number of degrees of freedom, and the hard scattering process can be generated quite efficiently. The initial state cascade is generated by going backwards from the hard scattering process towards the beam particles.

According to the CCFM equation the probability of finding a gluon in the proton depends on three variables, the momentum fraction $x$, the transverse momentum squared $k_{t}^{2}$ of the exchanged gluons and the scale $\bar{q}=x_{n} \sqrt{s \Xi}$, which is related to the maximum angle $\Xi$ allowed for any emission. To solve (8) the unintegrated parton distribution $\mathcal{A}\left(x, k_{t}, \bar{q}\right)$ has to be determined beforehand.

Given $\mathcal{A}\left(x, k_{t}, \bar{q}\right)$, the generation of a full hadronic event is separated into three steps, as implemented in the hadron level Monte Carlo program CASCADE:

- The hard scattering process is generated,

$$
\begin{aligned}
\sigma= & \int d k_{t 1}^{2} d k_{t 2}^{2} d x_{1} d x_{2} \mathcal{A}\left(x_{1}, k_{t 1}, \bar{q}\right) \mathcal{A}\left(x_{2}, k_{t 2}, \bar{q}\right) \\
& \hat{\sigma}\left(k_{1}+k_{2} \rightarrow X\right)
\end{aligned}
$$

with $k_{1}\left(k_{2}\right)$ being the momenta of the incoming partons to the subprocess $k_{1}+k_{2} \rightarrow X$ with $X$ being the final state. The definition of $\hat{\sigma}$ follows [10]. The available processes are shown in Table 2. The momenta of the incoming partons are given in Sudakov representation:

$$
\begin{aligned}
& k_{1}=x_{1} p^{(1)}+\bar{x}_{2} p^{(2)}+k_{t} \simeq x_{1} p^{(1)}+k_{t 1}, \\
& k_{2}=\bar{x}_{1} p^{(1)}+x_{2} p^{(2)}+k_{t} \simeq x_{2} p^{(2)}+k_{t 2},
\end{aligned}
$$

where the last expression comes from the high energy approximation $\left(x_{1,2} \ll 1\right)$, which then gives $-k^{2} \simeq k_{t}^{2}$.

- The initial state cascade is generated according to CCFM in a backward evolution approach.

- The hadronization is performed using the Lund string fragmentation implemented in Pythia/Jetset [11].

The parton virtuality enters the hard scattering process and also influences the kinematics of the produced particles $\left(Z_{0}, W\right.$, Higgs and quarks) and therefore the maximum angle allowed for any further emission in the initial state cascade. This virtuality is only known after the whole cascade has been generated, since it depends on the history of the parton evolution (as $\bar{x}$ in (15) may not be neglected for exact kinematics). In the evolution equations itself it does not enter, since there only the longitudinal energy fractions $z$ and the transverse momenta are involved. This problem can only approximately be overcome by using $k^{2}=k_{t}^{2} /(1-x)$ for the virtuality which is correct in the case of no further parton emission in the initial state.

The Monte Carlo program CASCADE can be used to generate unweighted full hadron level events, including initial state parton evolution according to the CCFM equation and the off-shell matrix elements for the hard scattering process. 
Table 1 Recommended CCFM unintegrated parton distribution functions included in

CASCADE. See also Sect. 5.5.5

\begin{tabular}{|c|c|c|c|c|c|c|c|}
\hline \multirow[t]{2}{*}{ Parton } & \multirow[t]{2}{*}{ uPDF set } & \multicolumn{2}{|c|}{$\begin{array}{l}x \mathcal{A}_{0}\left(x, k_{t}, \bar{q}\right) \\
=N x^{-B}(1-x)^{C}\end{array}$} & \multirow[t]{2}{*}{$\Lambda_{\mathrm{QCD}}^{(4)}$} & \multirow[t]{2}{*}{$k_{t}^{\text {cut }}$} & \multirow[t]{2}{*}{$Q_{0}$} & \multirow[t]{2}{*}{ Ref. } \\
\hline & & $\bar{B}$ & $C$ & & & & \\
\hline \multirow[t]{13}{*}{ Gluon } & set JS & 0 & 4 & 0.25 & 0.25 & 1.4 & [13] \\
\hline & set A0 & 0 & 4 & 0.25 & 1.3 & 1.3 & [13] \\
\hline & set $\mathrm{A} 0+$ & -0.01 & 4 & 0.25 & 1.3 & 1.3 & [13] \\
\hline & set A0- & -0.01 & 4 & 0.25 & 1.3 & 1.3 & [13] \\
\hline & set A1 & -0.1 & 4 & 0.25 & 1.3 & 1.3 & [13] \\
\hline & set B0 & 0 & 4 & 0.25 & 0.25 & 1.3 & [13] \\
\hline & set $\mathrm{B} 0+$ & 0.01 & 4 & 0.25 & 0.25 & 1.3 & [13] \\
\hline & set B0- & 0.01 & 4 & 0.25 & 0.25 & 1.3 & [13] \\
\hline & set B1 & -0.1 & 4 & 0.25 & 0.25 & 1.3 & [13] \\
\hline & set $\mathrm{C}$ & 0.25 & 4 & 0.13 & 1.1 & 1.1 & [14] \\
\hline & set 1 & 0 & 4 & 0.25 & 1.33 & 1.33 & [15] \\
\hline & set 2 & 0 & 4 & 0.25 & 1.18 & 1.18 & [15] \\
\hline & set 3 & 0 & 4 & 0.25 & 1.35 & 1.35 & [15] \\
\hline Quark & set A & - & - & 0.25 & 1.3 & 1.3 & \\
\hline
\end{tabular}

It is applicable for $p \bar{p}, p p$, photoproduction as well as for deep inelastic scattering. A discussion of the phenomenological applications of CASCADE can be found in [12].

The typical time needed to generate one event is similar to the time needed by standard Monte Carlo event generators like PYTHIA [11].

\subsection{The unintegrated parton density}

The CCFM unintegrated parton density $x \mathcal{A}\left(x, k_{t}, \bar{q}\right)$ can be obtained from a forward evolution procedure as implemented in SMALLX $[16,17]$ by a fit to the measured structure function $F_{2}$ as described i.e. in [5, 6]. From the initial parton distribution, which includes a Gaussian intrinsic $k_{t}$ distribution, a set of values $x$ and $k_{t}$ is obtained by evolving up to a given scale $\log \bar{q}$ using a forward evolution procedure. Technically the parton density is stored on a grid in $\log x, \log k_{t}$ and $\log \bar{q}$ and a linear interpolation is used to obtain the parton density for values in between the grid points. The data file (i.e. CCfm-xxxx. dat) containing the grid points is read in at the beginning of the program.

Several sets (J2003 set 1 - 3 [15] ( IGLU $=1001$ 1003), set A [13] IGLU $=1010-1013$ and set $\mathbf{B}$ [13] IGLU $=1020-1023$ ) of unintegrated gluon densities are available with the input parameters fitted to describe the structure function $F_{2}\left(x, Q^{2}\right)$ in the range $x<5 \times 10^{-3}$ and $Q^{2}>4.5 \mathrm{GeV}^{2}$ as measured at $\mathrm{H} 1[18,19]$ and ZEUS [20, 21]. Set JS [6] (IGLU=1) is fitted only to $F_{2}\left(x, Q^{2}\right)$ of [18]. The collinear cutoff $k_{t}^{\text {cut }}=Q_{0}$ which regulates the region of $z \rightarrow 1$, is applied both to the real emissions as well as inside the Sudakov form factor. In JS, J2003 set 1 - $\mathbf{3}$ and set A we have $k_{t}^{\text {cut }}=Q_{0}=1.3 \mathrm{GeV}$. Similarly, fits can be obtained using different values for the soft cut $k_{t}^{\text {cut }}=0.25 \mathrm{GeV}$, which are available in set $\mathbf{B}$. The set $\mathbf{C}($ IGLU $=1101)$ [14] uses the full splitting function, as described in (12), with a value for $\Lambda_{\mathrm{QCD}}^{(4)}=0.13 \mathrm{GeV}$. This set was obtained by fitting simultaneously the inclusive $F_{2}\left(x, Q^{2}\right)$ and jet measurements in DIS resulting in a changed intrinsic $k_{t}$ distribution. A CCFM parametrization of the valence quark distribution is available (using as starting distribution CTEQ 5 [22] and evolved with a splitting function $P_{q q}$ [3] including angular ordering of the emitted gluon). The available CCFM uPDF sets with the parameters of the starting distributions are listed in Table 1.

With the parameter IGLU also other unintegrated gluon densities are accessible: a simple numerical derivative of a standard integrated gluon density $\frac{d x g\left(x, Q^{2}\right)}{d Q^{2}}$ taken from [23] (IGLU=2), the one in the approach of Blümlein [24] and coded by $[25,26]$ (IGLU $=3)$, the unintegrated gluon density of $\mathrm{KMS}^{1}$ [27] (IGLU=4, stored in $\mathrm{kms}$. dat), the one of the saturation model by [28] (IGLU $=5$, i.e. parameter set including charm $)^{2}$ and the one of $\mathrm{KMR}^{3}$ [29] $($ IGLU $=6$, stored in $\mathrm{kmr}$. dat).

Initial state parton showers can be only generated for the CCFM unintegrated gluon density (with $I G L U=1$ and IGLU $=1001-1023,1101$ ). For all other sets only the cross section can be calculated without explicit inclusion of

\footnotetext{
${ }^{1} \mathrm{~A}$. Stasto kindly provided the program code.

${ }^{2}$ The values of $\alpha_{\mathrm{s}}$ and quark masses of [28] are not automatically used in the cross section calculation, but need to be set explicitly.

${ }^{3} \mathrm{M}$. Kimber kindly provided the program code.
} 
initial state parton showers, since the angular variable, essential for angular ordering in the initial state cascade, is not available in the uPDFs. However, the transverse momenta of the incoming partons are properly treated. Only the KMR set $($ IGLU $=6)$ provides a prescription for the emission of at most one additional gluon.

\section{Hard processes in CASCADE}

Different sets of hard processes applicable for lepto (photo) - and hadroproduction have been calculated and are implemented in CASCADE. The available processes are listed in Table 2.

\subsection{Lepto(photo)production}

CASCADE can be used to simulate leptoproduction events over the whole $Q^{2}$ range. By fixing the light quark masses to $m_{q}=0.25 \mathrm{GeV}$ and $\alpha_{s}$ for small $\mu$, the hard scattering matrix element remains finite over the full phase space. The total cross section is simulated by selecting IPRO $=10$ and $\mathrm{NFLAV}=4(5)$. With IPRO=10 light quarks $(u, d, s)$ are selected and with NFLAV $>3$ the program automatically includes heavy flavour production via the process $I P R O=11$ and IHFLA $=4$ up to IHFLA=NFLAV. The flag IRE1 indicates, whether beam 1 has a internal structure: $I R E 1=1$ is used to generate resolved photon events.

Table 2 Processes included in CASCADE. $Q$ stands for heavy quarks, $q$ for light quarks

\begin{tabular}{lll}
\hline Process & IPRO & Reference \\
\hline Leptoproduction & & \\
$\gamma^{*} g^{*} \rightarrow q \bar{q}$ & 10 & {$[10]$} \\
$\gamma^{*} g^{*} \rightarrow Q \bar{Q}$ & 11 & {$[10]$} \\
$\gamma^{*} g^{*} \rightarrow J / \psi g$ & 2 & {$[30-33]$} \\
\hline Hadroproduction & & \\
$g^{*} g^{*} \rightarrow q \bar{q}$ & 10 & {$[10]$} \\
$g^{*} g^{*} \rightarrow Q \bar{Q}$ & 11 & {$[10]$} \\
$g^{*} g^{*} \rightarrow J / \psi g$ & 2 & {$[33]$} \\
$g^{*} g^{*} \rightarrow \Upsilon g$ & 2 & {$[33]$} \\
$g^{*} g^{*} \rightarrow \chi c$ & 3 & {$[33]$} \\
$g^{*} g^{*} \rightarrow \chi b$ & 3 & {$[33]$} \\
$g^{*} g^{*} \rightarrow h^{0}$ & 102 & {$[34]$} \\
$g^{*} g^{*} \rightarrow Z Q \bar{Q}$ & 504 & {$[35,36]$} \\
$g^{*} g^{*} \rightarrow Z q \bar{q}$ & 503 & {$[35,36]$} \\
$g^{*} g^{*} \rightarrow W q_{i} Q_{j}$ & 514 & {$[35,36]$} \\
$g^{*} g^{*} \rightarrow W q_{i} q_{j}$ & 513 & {$[35,36]$} \\
$q g^{*} \rightarrow Z q$ & 501 & {$[37]$} \\
$q g^{*} \rightarrow q g$ & 10 & {$[38]$} \\
$g g^{*} \rightarrow g g$ & 10 & {$[38]$} \\
\hline & &
\end{tabular}

Heavy flavour production can be generated separately via $I P R O=11$. The value of IHFLA determines the heavy flavour to be generated.

The matrix element for $\gamma g^{*} \rightarrow J / \psi(\Upsilon) g$ calculated in [31-33] is available for quasi-real $\gamma$ 's via the process $I P R O=2$. The flavour of the Onium is selected via IHFLA, i.e. IHFLA $=4$ for $J / \psi$ and IHFLA $=5$ for $\Upsilon$. The matrixelement including $J / \psi(\Upsilon)$ polarization and subsequent leptonic decay can be selected with IPSIPOL $=1$.

CASCADE can be used to simulate real photoproduction events by using $\mathrm{KBE} 1=22$. The same options as for leptoproduction are available. Resolved photon events can be generated with $I R E 1=1$.

\subsection{Hadroproduction}

The hadroproduction processes available are listed in Table 2. The flavour code for beam 1 (2) can be chosen as $\mathrm{KBE} 1=2212$ for proton or $\mathrm{KBE} 1=-2212$ for anti-proton, for beam $2 \mathrm{KBE} 2$ is changed accordingly.

CASCADE can be used to simulate heavy quark production in $p p$ or $p \bar{p}$ collisions $\left(g^{*} g^{*} \rightarrow Q \bar{Q}\right.$ I $\mathrm{PRO}=11$ for heavy flavour production, and $I H F L A=4$ ( 5 ) for charm (bottom) quarks), but also for light quarks with IPRO $=10$. The matrix element for $g^{*} g^{*} \rightarrow J / \psi g$ calculated in [3133 ] is available via the process $I P R O=2$. The matrixelement including $J / \psi$ polarization and subsequent leptonic decay can be selected with IPSIPOL $=1$. The process $g^{*} g^{*} \rightarrow \chi$ is available with $I P R O=3$ including all three $\chi$ states with appropriate spin and angular momentum. The flavour of the Onium is selected via IHFLA, i.e. IHFLA $=4$ for $J / \psi\left(\chi_{c}\right)$ and IHFLA $=5$ for $\Upsilon\left(\chi_{b}\right)$.

Apart from the ground states, the $2 S$ and $3 S$ states for the $\Upsilon$ family and the $2 S$ state for the $J / \psi$ family can be generated vis $i 23 s=2,3$. The wavefunction is calculated from the corresponding leptonic decay width, as done for the $1 S$ state. The wavefunction for the $1 P$ and $2 P$ states are taken from [42]. Also the $2 P$ states of $\chi_{b}$ with subsequent decay into $\Upsilon(2 S)$ are included. The decay of the $\Upsilon(n S)$ and $J / \psi(2 S)$ as well as the $\chi$ states is performed within the PYTHIA framework by adding new particles and decay modes via PYUPDA. The flavor codes used are given in Table 3 .

The process $g^{*} g^{*} \rightarrow h^{0}$ with the matrix element calculated in [34] is available via $I P R O=102$, the Higgs mass can be selected via PMAS (25).

The process $g^{*} g^{*} \rightarrow Z q \bar{q}$, calculated in [35, 36], is available via $I P R O=503$ for light quarks and $I P R O=504$ for the heavy quarks. The flavor of the heavy quark is selected via $\operatorname{IHFLA}=4 \quad(5,6)$ for charm (bottom, top). The process $g^{*} g^{*} \rightarrow W q_{i} q_{j}$, calculated in $[35,36]$, is available via $I P R O=513$ for light quarks and $I P R O=514$ for heavy quarks, where the flavour of the heaviest quark is defined 
Table 3 Flavor codes used to define the excited states of vectormesons

Vectormeson

$J / \psi(1 S)$

$J / \psi(2 S)$

$\Upsilon(1 S)$

$\Upsilon(2 S)$

$\Upsilon(3 S)$

$\chi_{b 0}(1 P)$

$\chi_{b 1}(1 P)$

$\chi_{b 2}(1 P)$

$\chi_{b 0}(2 P)$

$\chi_{b 1}(2 P)$

$\chi_{b 2}(2 P)$

by $I H F L A=3, \quad(4,5)$. Which of the quarks are produced depends on the charge of the $W$ which is randomly selected.

The processes $g^{*} g \rightarrow g g$ and $g^{*} q \rightarrow g q$ [38] are included via process $I P R O=10$. The individual processes can be selected for $g^{*} g^{*} \rightarrow q \bar{q}$ via IRPA $=1, g^{*} g \rightarrow g g$ via $\mathrm{IRPB}=1$ and $g^{*} q \rightarrow g q$ via IRPC $=1$. Note that here one of the partons is treated on-shell. For the quarks the unintegrated quark distribution (for valence quarks) is used.

The process $q g^{*} \rightarrow Z q$, calculated in [35, 37], is available via $I P R O=501$, using the unintegrated (valence) quark distribution for the on-shell quark.

\section{$4.3 \alpha_{s}$ and the choice of scales}

The strong coupling $\alpha_{s}$ is calculated via the PYTHIA [11] subroutine PYALPS. Maximal and minimal number of flavours used in $\alpha_{s}$ are set by MSTU (113) and MSTU (114), $\Lambda_{\mathrm{QCD}}=\mathrm{PARU}$ (112) with respect to the number of flavours given in MSTU (112) and stored in the PYTHIA common block COMMON/PYDAT1/. In the initial state cascade according to CCFM, the transverse momenta of the $t$-channel gluons are allowed to perform a random walk for small $z$ values and $k_{t}$ can become very small. In the $1 / z$ part of the splitting function we use $\mu=k_{t}$ as the scale in $\alpha_{s}(\mu)$ and in the $1 /(1-z)$ part $\mu=p_{t}$ is used. In addition we require $\mu>Q_{0}$, resulting in $\alpha_{s}\left(\mu>Q_{0}\right)<0.6$.

The scale $\mu$, which is used in $\alpha_{s}$ in the hard scattering matrix element, can be changed with the parameter IQ2, the default choice is $\mu^{2}=p_{t}^{2}$.

The renormalization scale dependence of the final cross section can be estimated by changing the scale used in $\alpha_{s}$ in the off-shell matrix element. Since here we are using the LO $\alpha_{s}$ matrix elements, any scale variation will change the cross section. In order to obtain a reasonable result, the uPDF was fitted to describe $F_{2}$ by varying the scale $\mu_{r}$. The set A0-, B0- correspond to a scale $\mu_{r}=0.5 p_{t}$ whereas set $\mathrm{A0}+, \mathbf{B} 0+$ correspond to a scale $\mu_{r}=2 p_{t}$.
In order to investigate the uncertainties coming from the specific choice of the evolution scale, another definition is applied, relating the factorization scale only to the quark (or anti-quark): $\mu_{f}=\frac{p_{t}}{1-z}$ with $p_{t}$ being the transverse momentum of the quark (anti-quark) and $z=\frac{\tilde{k}_{t}}{y x_{g} s}$. The set A1, B1 correspond to a scale $\mu_{f}=\frac{p_{t}}{1-z}$. In the PDF set $\mathbf{C} \Lambda_{\mathrm{QCD}}^{(4)}$ was fixed to $\Lambda_{\mathrm{QCD}}^{(4)}=0.13 \mathrm{GeV}$.

\subsection{Quark masses}

The quark mass for light quarks $(u, d, s)$ is fixed to $m_{q}=$ $0.25 \mathrm{GeV}$. This, together with the treatment of $\alpha_{s}$ at small scales $\mu$, gives also a reasonable total cross-section for photoproduction at HERA energies. The masses for heavy quarks are given by the JETSET/PYTHIA defaults $\left(m_{c}=1.5\right.$ $\mathrm{GeV}, m_{b}=4.8 \mathrm{GeV}$ ) and can be changed according to the PYTHIA prescription.

\subsection{Initial and final state parton showers}

Initial state parton showers are generated in a backward evolution approach described in detail in $[5,6]$. The initial state parton shower consists only of gluon branchings and is generated in an angular ordered region in the laboratory frame. The gluons emitted in the initial state can undergo further timelike branchings. The maximum timelike mass $m_{\max }$ is calculated using the angular constraint. With this mass, the parton which can further undergo a timelike branching is boosted to its rest frame with $m_{\max }$ but keeping the original energy. The timelike branching is performed with the PYTHIA routine PYSHOW. After successful timelike branching, the proper mass is associated to the parton and the kinematics are calculated appropriately. Gluon radiation from the valence quarks is also included.

All parameters (like the scale $\mu$ in $\alpha_{s}$, the collinear cutoff $k_{t}^{\text {cut }}=Q_{0}$ ) for the initial state cascade are fixed from the determination of the unintegrated gluon density. The transverse momenta of the partons which enter the hard scattering matrix element are already generated in the beginning and are not changed during the whole initial and final state parton showering.

The final state parton shower uses the parton shower routine PYSHOW of PYTHIA with the default scale $\mu^{2}=$ $2 \cdot\left(m_{1 \perp}^{2}+m_{2 \perp}^{2}\right)(\mathrm{IFIN}=1)$, with $m_{1(2) \perp}$ being the transverse mass of the hard parton 1(2). Other choices are possible: $\mu^{2}=\hat{s}(\operatorname{IFIN}=2)$ and $\mu^{2}=2 \cdot\left(m_{1}^{2}+m_{2}^{2}\right)($ IFIN=3). In addition a scale factor can be applied: $\operatorname{SCAF} \times \mu^{2}$ (default: $\mathrm{SCAF}=1)$.

\subsection{Remnant treatment}

In CASCADE version 1 the proton remnant was built in subroutine CAREMN, which is a slightly modified version of 
the PYTHIA/LEPTO subroutine PYREMN. No intrinsic transverse momentum, in addition to the transverse momentum from the initial state cascade, was included.

From version 2.0 on the proton remnant can be generated directly via PYTHIA by selecting ILHA=10 (which is now the default). The structure of the event record is then identical to the one obtained from a standard PYTHIA run.

\subsection{Hadronization}

In CASCADE version 1 the hadronization was done exclusively by PYTHIA. From version 2 onwards, events can be written into a file (via switch $I L H A=1$ ) according the LHA Accord [39], which can be read by any hadronization program (like PYTHIA or HERWIG), generating the remnants and performing the hadronization. With ILHA $=10$ the hadronization is performed within PYTHIA. The old CASCADE format is obtained with $I L H A=0$. Please note, that top decays can only be simulated properly within the PYTHIA fragmentation and are therefore available only with $I L H A=10$.

\section{Description of the program components}

In CASCADE all variables are declared as Double PreCision. The Lund string model is used for hadronization as implemented in PYTHIA [11]. The final state QCD radiation is performed via PYSHOW from PYTHIA. The treatment of the proton remnant follows very closely the ones in LEPTO [40] for the leptoproduction case and the one in PYTHIA for the proton-proton case. However slight modifications were needed to adapt to the cascade treatment here.

The unintegrated gluon density is stored on data files (ccfm.dat, kms. dat, kmr. dat), and is read in at the beginning of the program.

The program has to be compiled and linked together with PYThia 6, to ensure that the double precision code of JETSET is loaded.

\subsection{Random number generator}

Since the variables are declared as double precision, also a double precision random number generator has to be used to avoid any bias. The function DCASRN gives a single random number, the function DCASRNV returns an array of length LEN of random numbers. The default random number generator is RANLUX (called in DCASRN and DCASRNV) The source code of RANLUX (extracted from CERNLIB) is included in the distribution. The user can change this to any preferred Double Precision random number generator.

5.2 Integration and event generation

The integration of the total cross section and the generation of unweighted events is performed with the help of BASES/SPRING [41], which is included in the distribution package.

\subsection{Program history}

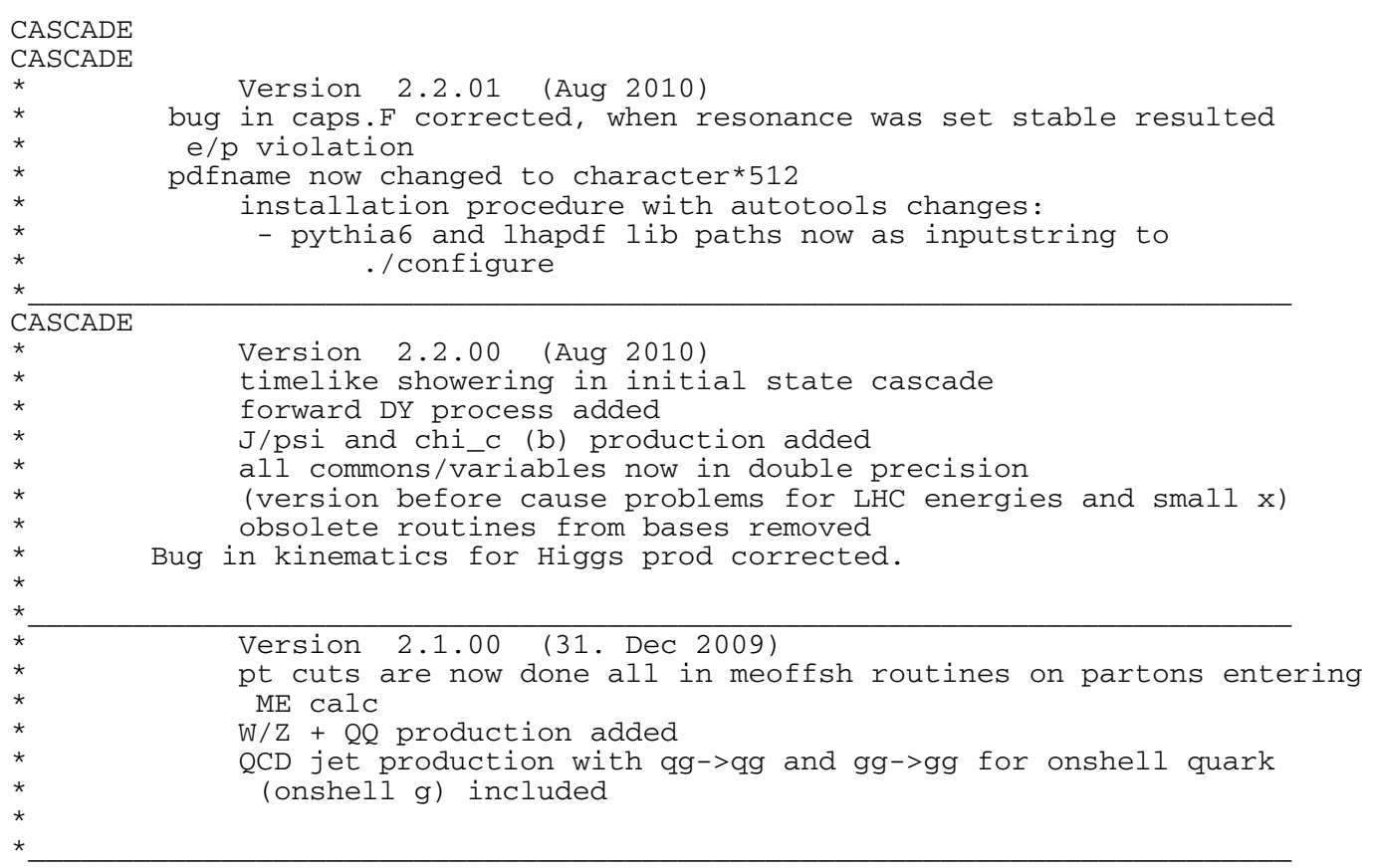




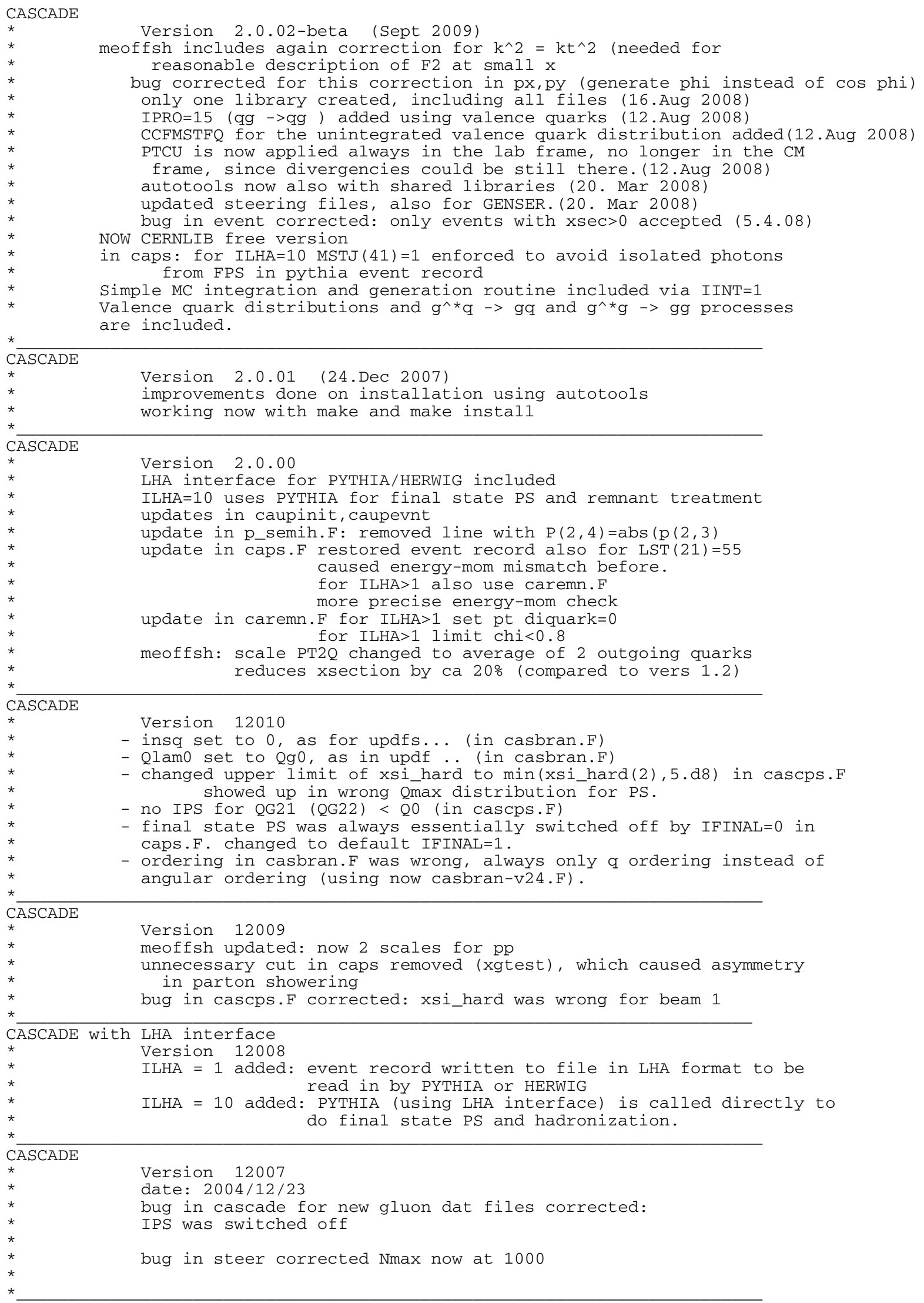




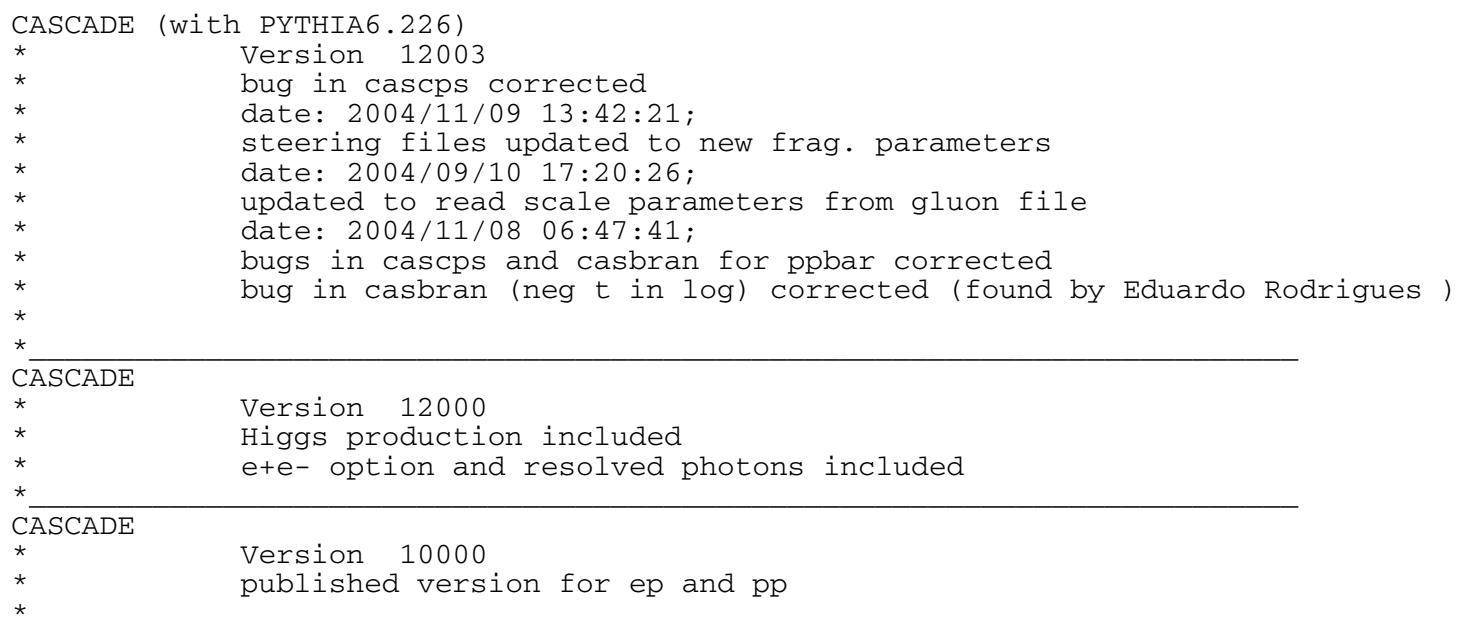

\subsection{Subroutines and functions}

The source code of CASCADE and this manual can be found under: http://www.desy.de/ jung/cascade/

CAMAIN main program.

CASINI to initialises the program.

CASCADE to perform integration of the cross section. This routine has to be called before event generation can start.

CAEND to print the cross section and the number of events.

CAUNIGLU $(K F, X, K T, P, X P Q)$ to extract the unintegrated gluon density $x \mathcal{A}\left(x, k_{t}, \bar{q}\right)$ for a proton with $\mathrm{KF}=2212$, as a function of $x=\mathrm{X}, k_{t}^{2}=\mathrm{KT}$ and $\bar{q}=\mathrm{P}$. The gluon density is returned in XPQ $(0)$, where $X P Q$ is an array with XPQ $(-6: 6)$.

EVENT to perform the event generation.

ALPHAS (RQ) to give $\alpha_{s}(\mu)$ with $\mu=\mathrm{RQ}$.

PARTI to give initial particle and parton momenta.

FXN1 to call routines for selected processes: XSEC1.

CUTG ( IPRO) to cut on $p_{t}$ for $2 \rightarrow 2$ process in integration and event generation.

MEOFFSH matrix element for $\gamma^{*} g^{*} \rightarrow q \bar{q}$ and $g^{*} g^{*} \rightarrow$ $q \bar{q}$ including quark masses. $q$ can be light or heavy quarks. MEHIGGS matrix element for $\gamma^{*} g^{*} \rightarrow h^{0}$.

$\mathrm{DOT}$ (A, B) four-vector dot product of $A$ and $B$.

DOT1 ( $I, J$ ) four-vector dot product of vectors $I$ and $J$ in PYJETS common.

PHASE to generate momenta of final partons in a $2 \rightarrow 2$ subprocess according to phase space.

P_SEMIH to generate kinematics and the event record for $e p, \gamma p$ and $p \bar{p}$ processes.

CAREMN ( IPU1, IPU2) to generate the beam remnants. Copied from LEPTO 6.1 [40] and updated for the use in CASCADE.

CASPLI ( $K F, K P A, K F S P, K F C H)$ to give the spectator $\mathrm{KFSP}$ and $\mathrm{KFCH}$ partons when a parton KPA is removed from particle KF. Copied from LEPTO 6.1 [40] and updated for the use CASCADE.
CAPS to generate color flow for all processes and prepare for initial and final state parton showers.

CASCPS (IPU1, IPU2) to generate initial state radiation.

COLORFLOW to generate color configuration for $g^{*} g \rightarrow$ $g g$ and $g^{*} q \rightarrow q g$ processes.

GADAP Gaussian integration routine for 1-dim and 2-dim integration. Copied from LEPTO 6.1 [40].

\subsection{Parameter switches}

BASES/SPRING integration procedure.

NCAL : $(D:=20000) \mathrm{Nr}$ of calls per iteration for bases.

ACC1 : (D :=1) relative precision (in \%) for grid optimization.

ACC2 : $(\mathrm{D}:=0.5)$ relative precision (in \%) for integration. Event record output.

ILHA : (D :=0) output in LHA accord [39] format $=0:$ CASCADE type output of event record

$=1$ : output of event record to be read in by fragmentation programs

$=10$ : use LHA format to produce remnant and fragmentation in PYTHIA style.

\subsubsection{Parameters for kinematics}

PBE1: (D := -30) momentum $p[\mathrm{GeV} / c]$ of incoming hadron 1 (/INPU/).

KBE1 : Lund flavour code of incoming hadron $1(\mathrm{KBE} 1=11$ for electrons, $\mathrm{KBE} 1=22$ for photons, $\mathrm{KBE} 1=2212$ for protons).

IRE1 : hadron/lepton 1 has a structure $(\operatorname{IRE} 1=1)$ or interacts directly with the target (IRE $1=0$ for a DIS electron) PBE2 : (D := 820 momentum $p[\mathrm{GeV} / c]$ of incoming proton ( / INPU /).

$\mathrm{KBE} 2$ : Lund flavour code of incoming hadron $2(\mathrm{KBE} 2=$ 11 for electrons, $\mathrm{KBE} 2=22$ for photons, $\mathrm{KBE} 2=2212$ for protons). 
IRE2 : hadron/lepton 2 has a structure $(\operatorname{IRE} 2=1)$ or interacts directly with the target (IRE2 $=0$ for a DIS electron).

NFLAV (D :=5) number of active flavours, can be set by user (/CALUCO /).

\subsubsection{Parameters specific for leptoproduction}

QMI : (D :=5.0) (/VALUES $/)$ minimum $Q^{2}$ to be generated.

QMA: $\left(D:=10^{8}\right)(/$ VALUES $/)$ maximum $Q^{2}$ to be generated.

YMI : $(\mathrm{D}:=0.0)(/$ VALUES $/)$ minimum $y$ to be generated.

YMA : $(\mathrm{D}:=1.0)(/$ VALUES $/)$ maximum $y$ to be generated.

THEMA, THEMI (D: THEMA $=180$., THEMI $=0$ ) $\max$ imum and minimum scattering angle $\theta$ of the electron (/CAELEC/).

\subsubsection{Parameters for hard subprocess selection}

IPRO: $(\mathrm{D}:=10)(/ \mathrm{CAPAR} 1 /)$ selects hard subprocess to be generated.

$=2: \gamma g^{*} \rightarrow J / \psi(\Upsilon) g, g^{*} g^{*} \rightarrow J / \psi(\Upsilon) g$.

$=3: g^{*} g^{*} \rightarrow \chi_{c}(b)$.

$=10: \gamma^{*} g^{*} \rightarrow q \bar{q}, g^{*} g^{*} \rightarrow q \bar{q}, g^{*} g \rightarrow g g$ and $g^{*} q \rightarrow g q$ for light quarks.

$=11: \gamma^{*} g^{*} \rightarrow Q \bar{Q}$ or $g * g^{*} \rightarrow Q \bar{Q}$ for heavy quarks.

$=102: g^{*} g^{*} \rightarrow h^{0}$ for Higgs production in hadron-hadron collisions.

$=501: q g^{*} \rightarrow Z q$ for $Z+$ jet production in hadron-hadron collisions.

$=503: g * g^{*} \rightarrow Z q \bar{q}$ for $Z+$ jet production in hadronhadron collisions.

$=504: g * g^{*} \rightarrow Z Q \bar{Q}$ The flavour index of the heaviest quark is selected via IHFLA

$=513: g * g^{*} \rightarrow W q_{i} q_{j}$ for $W+$ jet production in hadronhadron collisions.

$=514: g * g^{*} \rightarrow W q_{i} Q_{j}$ for $W+$ jet production in hadronhadron collisions.

IRPA: $=1 g^{*} g^{*} \rightarrow q \bar{q}$ switched on for IPRO $=10$.

IRPB : $=1 g^{*} g \rightarrow g g$ switched on for IPRO $=10$.

IRPC : $=1 g^{*} q \rightarrow g q$ switched on for IPRO $=10$.

IHFLA: $(\mathrm{D}:=4)=4$ flavor of heavy quark produced (in

IPRO $=10$, IPRO $=504$ and IPRO=514).

IPSIPOL: $(\mathrm{D}:=0)(/ \mathrm{JPSI} /)$

$=1$ use matrixelement including $J / \psi(\Upsilon)$ polarization and subsequent leptonic decay for IPRO $=2$.

I23s: $(\mathrm{D}:=0)(/ \mathrm{JPSI} /)$

$=0$ use $1 S$ and $1 P$ sates for vectormeson production via IPRO $=2,3$

$=2$ use $2 S$ and $2 P$ states

$=3$ use $3 S$ states.

PT2CUT (IPRO) : $(\mathrm{D}=0.0)$ minimum $\hat{p}_{\perp}^{2}$ for process IPRO (/CAPTCUT/).
5.5.4 Parameters for parton shower and fragmentation

NFRAG: (D :=1) switch for fragmentation (/CAINPU/).

$=0$ : off

$=1$ : on.

IFPS : (D :=3) switch for parton shower (/CAINPU/).

$=0$ : off

$=1$ : initial state

$=2$ : final state

$=3$ : initial and final state.

ITIMSHR: $(\mathrm{D}:=1)$

$=0$ : no shower of time like partons

$=1$ : time like partons may shower.

ICCFM : $(\mathrm{D}:=1)$

$=1$ : CCFM evolution (all loops)

$=0$ : DGLAP type evolution (one loop).

IFIN $(\mathrm{D}:=1)$ scale switch for final state parton shower

$=1: \mu^{2}=2\left(m_{1 t}^{2}+m_{2 t}^{2}\right)$

$=2: \mu^{2}=\hat{s}$

$=3: \mu^{2}=2\left(m_{1}^{2}+m_{2}^{2}\right)$.

SCAF $(D:=1$.) scale factor for final state parton shower.

\subsubsection{Parameters for structure functions, $\alpha_{s}$ and scales}

IRUNAEM : $(\mathrm{D}:=0)(/ \mathrm{CAPAR} 1 /)$ select running of $\alpha_{e m}\left(Q^{2}\right)$.

$=0$ : no running of $\alpha_{e m}\left(Q^{2}\right)$

$=1$ : running of $\alpha_{e m}\left(Q^{2}\right)$.

IRUNA : (D :=1) switch for running $\alpha_{s}$.

$=0$ : fixed $\alpha_{s}=0.3$

$=1$ : running $\alpha_{s}\left(\mu^{2}\right)$.

IQ2: $(\mathrm{D}:=3)$ select scale $\mu^{2}$ for $\alpha_{s}\left(\mu^{2}\right)$.

$=1: \mu^{2}=4 \cdot m_{q}^{2}$ (use only for heavy quarks!)

$=2: \mu^{2}=\hat{s}$ (use only for heavy quarks!)

$=3: \mu^{2}=4 \cdot m^{2}+p_{\perp}^{2}$

$=4: \mu^{2}=Q^{2}$

$=5: \mu^{2}=Q^{2}+p_{\perp}^{2}+4 \cdot m^{2}$.

IGLU: (D := 1010) select unintegrated gluon density (/GLUON/).

Note that initial state parton showers not possible for IGLU $=2,3,4,5$

$=1$ : CCFM old set JS2001 [6]

$=1001$ : CCFM J2003 set 1 [15]

$=1002:$ CCFM J2003 set 2 [15]

$=1003:$ CCFM J2003 set 3 [15]

=1010: CCFM set A0 [13]

$=1011:$ CCFM set A0+ [13]

$=1012:$ CCFM set A0- [13]

$=1013:$ CCFM set A1 [13]

$=1020:$ CCFM set B0 [13]

$=1021:$ CCFM set B0+ [13]

$=1022:$ CCFM set B0- [13]

$=1023$ : CCFM set B1 [13] 
$=1101:$ CCFM set C [14]

$=2:$ derivative of GRV $[23] \frac{d x g\left(x, Q^{2}\right)}{d Q^{2}}$

$=3$ : approach of Blümlein [24]

$=4:$ KMS [27] $(\mathrm{kms} \cdot$ dat $)$

$=5$ : saturation model [28]

$=6:$ KMR $[29](\mathrm{kmr} \cdot$ dat $)$.

\subsubsection{Accessing information}

AVGI integrated cross section (/CAEFFIC/).

SD standard deviation of integrated cross section (/CAEFFIC/).

SSS squared center of mass energy $s$ (/CAPARTON/).

PBEAM energy momentum vector of beam particles (/CABEAM/).

KBEAM flavour code of beam particles (/CABEAM/).

Q2 in leptoproduction: actual $Q^{2}$ of $\gamma$ (/CAPAR4/).

YY negative light-cone momentum fraction of parton 1 $\left(\gamma^{*}, g^{*}\right)(/$ CASGKI $/)$.

YY_BAR positive light-cone momentum fraction parton 1 $\left(\gamma^{*}, g^{*}\right)(/$ CASGKI $/)$.

XG positive light-cone momentum fraction of parton $2\left(g^{*}\right)$ (/CASGKI/).

XG_BAR negative light-cone momentum fraction of parton $2\left(g^{*}\right)(/ \mathrm{CASGKI} /)$.

KT2_1,KT2_2 transverse momenta squared $k_{t 1(2)}^{2}\left[\mathrm{GeV}^{2}\right]$ of partons 1(2) which enter to the matrix element.

YMAX, YMIN actual upper and lower limits for $y=Y Y$ (/CAPAR5/).

Q2MAX, Q2MIN actual upper and lower limits for $Q^{2}$ (corresponding to KT2_1) of $\gamma$ (/CAPAR5/).

XMAX, XMIN upper and lower limits for $x$ (/CAPAR5/).

$\mathrm{AM}(18)$ vector of masses of final state particles of hard interaction (/CAPAR3/).

SHAT invariant mass $\hat{s}\left[\mathrm{GeV}^{2}\right]$ of hard subprocess (/CA$\operatorname{PAR} 5 /$ ).

NIA1, NIA2 position of partons in hard interaction in PYJETS event record (/CAHARD/).

$\mathrm{NF} 1, \mathrm{NF} 2$ first and last position final partons/particles of hard interaction in PYJETS (/CAHARD/).

Q2Q hard scattering scale $\mu^{2}$ used in $\alpha_{s}$ and structure functions (/CAPAR4 /).

ALPHS actual $\alpha_{S}(/$ CAPAR2 /).

ALPH $\alpha_{e m}(/$ CAPAR2 /).

NIN number of trials for event generation (/CAEFFIC/).

NOUT number of successful generated events (/CAEFFIC/).

\subsection{List of COMMON blocks}

\author{
COMMON / CABEAM / PBEAM $(2,5), \operatorname{KBEAM}(2,5)$, \\ $\operatorname{KINT}(2,5)$ \\ COMMON/CAHARD/NIA1, NIA2 , NIR2 , NF1, NF2 \\ COMMON / CAHFLAV / IHFLA
}

COMMON/CAINPU / PLEPIN, PPIN, NFRAG, ILEPTO, IFPS, IHF, INTER, ISEMIH

COMMON/CALUCO/KE, KP , KEB , KPH , KGL , KPA, NFLAV

COMMON/CAEFFIC / AVGI , SD , NIN , NOUT

COMMON/CAELEC/THEMA, THEMI

COMMON/CAGLUON/IGLU

COMMON/CAPAR1/IPRO, IRUNA, IQ2, IRUNAEM

COMMON / CAPAR2 / ALPHS , PI , ALPH , IWEI

COMMON/CAPAR3 / AM (18), PCM $(4,18)$

COMMON/CAPAR4 / Q2, Q2Q

COMMON/CAPAR5 / SHAT, YMAX, YMIN, Q2MAX, Q2MIN, XMAX, XMIN

COMMON/CAPAR6/LST ( 30 ), IRES ( 2)

COMMON/CAPARTON/SSS, CM (4) , DBCMS (4)

COMMON/CAPTCUT / PT2CUT ( 20 )

COMMON/CASKIN/YY, YY_BAR, XG , XG_BAR, KT2_1, KT2_2, PT2H, SHH

COMMON/VALUES/QMI , YMI , QMA, YMA

\section{Example Program}

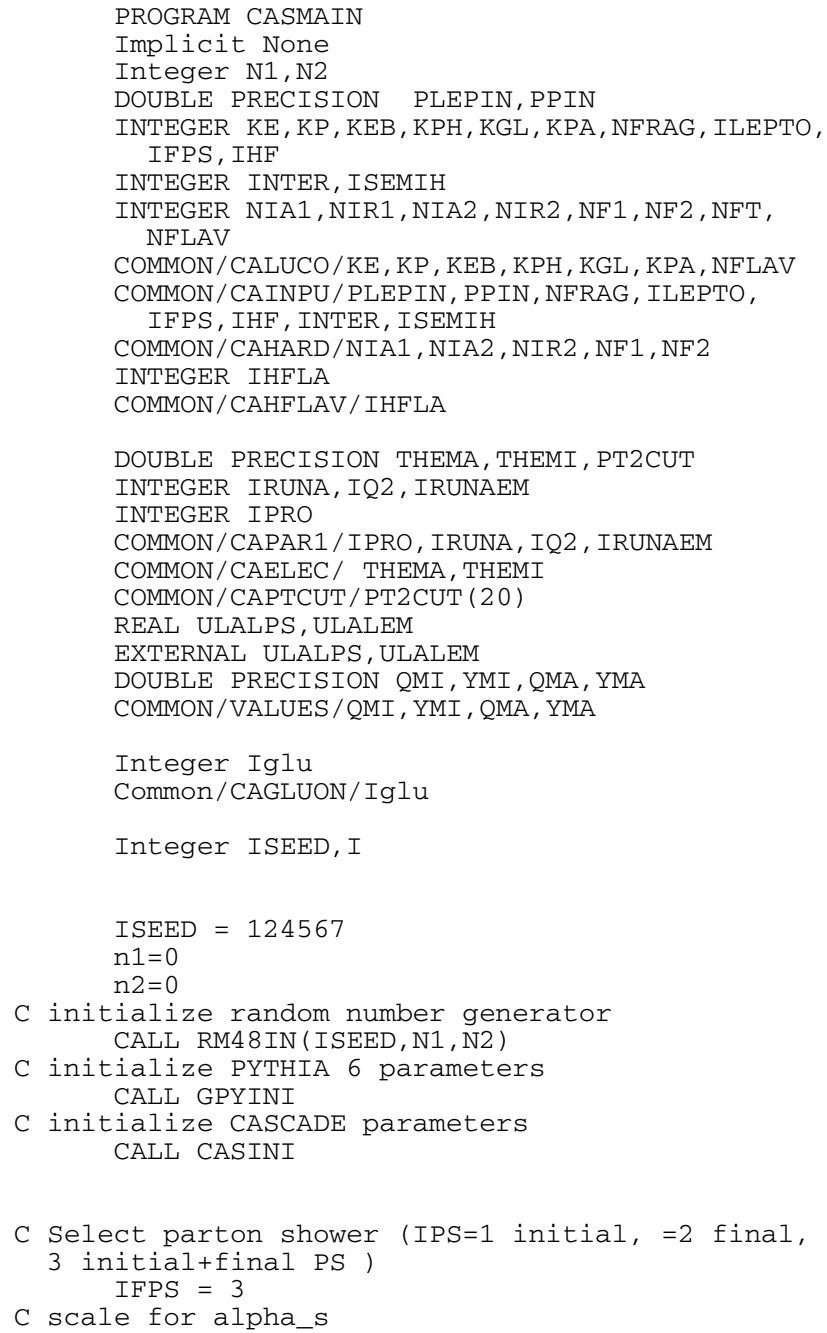


C IQ2 $=1 \mathrm{mu}^{\wedge} 2=4 \mathrm{~m} \_\mathrm{q}^{\wedge} 2 \quad\left(\mathrm{~m} \_\mathrm{q}=\right.$ light quark or heavy quark depending on IPRO)

$\mathrm{C}$ IO2 $=2 \mathrm{mu}^{\wedge} 2$ = shat

C IQ2 $=3 \mathrm{mu}^{\wedge} 2=4 \mathrm{~m} \_\mathrm{q}^{\wedge} 2+\mathrm{pt}^{\wedge} 2 \quad\left(\mathrm{~m} \_\mathrm{q}=\right.$ light quark or heavy quark depending on IPRO)

C IQ2 $=4 \mathrm{mu} \wedge 2=\mathrm{q}^{\wedge} 2$ ( $\mathrm{q}^{\wedge} 2$ of virtual photon)

C IQ2 $2=5 \mathrm{mu}^{\wedge} 2=\mathrm{q}^{\wedge} 2+\mathrm{pt}^{\wedge} 2+4 \mathrm{~m}-\mathrm{q}^{\wedge} 2\left(\mathrm{q}^{\wedge} 2\right.$ of virtual photon) $I Q 2=3$

C select process (IPRO=10 for light quarks, IPRO=11 for heavy quarks) $I P R O=10$

$C$ total number of flavours involved NFLAV $=4$

$C$ select unintegrated gluon density $(D=1)$ $\operatorname{Iglu}=1$

C minimum $Q^{\wedge} 2$ of electron to be generated $\mathrm{QMI}=0.5 \mathrm{~d} 0$

C maximum $Q^{\wedge} 2$ of electron to be generated $\mathrm{QMA}=10 \mathrm{D} 8$

$C$ minimum y of electron to be generated YMI $=0.0 \mathrm{do}$

$C$ minimum $y$ of electron to be generated $\mathrm{YMA}=1.0 \mathrm{~d} 0$

$C$ maximum theta angle of scattered electron THEMA $=180.0 \mathrm{DO}$

$C$ minimum theta angle of scattered electron THEMI $=0.0 \mathrm{DO}$

C momentum of beam 1 (electron, proton, antiproton) PLEPIN $=-27.5$

C Lund flavour code for beam 1 (electron=11, photon $=22$, proton=2212, antiproton $=-2212$ ) $\mathrm{KE}=11$

C momentum of beam 2 (proton) PPIN $=820$.

C perform fragmentation $\mathrm{NFRAG}=0 / 1$ NFRAG $=1$

c for $\mathrm{IPRO}=11$ which flavour is produced IHFLA $=4$

C start integration of $\mathrm{x}$-section

C

CALL CASCADE

C Print out result of integration of $\mathrm{x}$-section C

CALL CAEND (1)

C Start event loop

Do $I=1,100$

C generate an event Enddo

CALL EVENT

C Print out of generated events summary

CALL CAEND (20)

STOP

END

\section{Program Installation}

CASCADE now follows the standard AUTOMAKE convention. To install the program, do the following

1) Get the source

tar xvfz cascade-Xxxx.tar.gz

cd cascade-XXXX

2) Generate the Makefiles (do not use shared

libraries)

-/configure --disable-shared --prefix=install-path

--with-pythia6="pythia_path"

--with-1hapdf="1hapdflib_path" with (as example)

pythia_path=/afs/desy.de/group/alliance/mcg/

public/MCGenerators/pythia6/422/i586_rhel40

lhapdflib_path=/afs/desy.de/group/alliance/mcg/

public/MCGenerators/lhapdf/5.8.1/i586_rhel40

3) Compile the binary

make

4) Install the executable and PDF files make install

4) The executable is in bin

set the path for the updf data files, if different from the default (for example)

export PDFPATH=/Users/jung/jung/cvs/cascade2 /

cascade-2.2.0/share

run it with:

cascade $<$ steer_pp-bottom

Acknowledgements We are very grateful to B. Webber for providing us with the SMALLX code, which was the basis for the CASCADE Monte Carlo generator. We are very grateful also to G. Ingelman and T. Sjöstrand for many discussions and for their courtesy to let us use their code for proton remnant treatment. One of us (H.J.) enjoyed very much the collaboration with G. Salam and his patience and help in all different kinds of discussions concerning CCFM and a backward evolution approach. We have enjoyed and learned a lot from the discussions with B. Andersson, G. Gustafson, L. Jönsson, H. Kharraziha and L. Lönnblad during several years. Some of us (S.B., H.J., A.L. and N.Z.) are very grateful to DESY Directorate for the support in the framework of Moscow-DESY project on Monte-Carlo implementation for HERA - LHC.

Open Access This article is distributed under the terms of the Creative Commons Attribution Noncommercial License which permits any noncommercial use, distribution, and reproduction in any medium, provided the original author(s) and source are credited.

\section{References}

1. M. Ciafaloni, Nucl. Phys. B 296, 49 (1988)

2. S. Catani, F. Fiorani, G. Marchesini, Phys. Lett. B 234, 339 (1990)

3. S. Catani, F. Fiorani, G. Marchesini, Nucl. Phys. B 336, 18 (1990)

4. G. Marchesini, Nucl. Phys. B 445, 49 (1995)

5. H. Jung, Ccfm prediction on forward jets and $f_{2}$ : parton level predictions and a new hadron level Monte Carlo generator CASCADE, in Proceedings of the Workshop on Monte Carlo Generators for HERA Physics, ed. by A. Doyle, G. Grindhammer, G. Ingelman, H. Jung (DESY, Hamburg, 1999), p. 75. arXiv:Hep-ph/9908497

6. H. Jung, G. Salam, Eur. Phys. J. C 19, 351 (2001) arXiv: hep-ph/0012143

7. B. Andersson, et al. (Small x Collaboration), Eur. Phys. J. C 25, 77 (2002). arXiv:Hep-ph/0204115

8. T. Sjöstrand, Phys. Lett. B 157, 321 (1985)

9. M. Bengtsson, T. Sjöstrand, Z. Phys. C 37, 465 (1988)

10. S. Catani, M. Ciafaloni, F. Hautmann, Nucl. Phys. B 366, 135 (1991)

11. T. Sjostrand et al., Comput. Phys. Commun. 135, 238 (2001). arXiv:Hep-ph/0010017

12. F. Hautmann, H. Jung, J. High Energy Phys. 10, 113 (2008). arXiv:0805.1049v3 [hep-ph]

13. H. Jung, Un-integrated parton density functions in ccfm, April 2004. DIS 2004, Strbské Pleso, Slovakia. arXiv:hep-ph/0411287 
14. M. Hansson, H. Jung, Towards precision determination of updfs, 2007. DIS 2007, Munich. arXiv:0707.4276 [hep-ph]

15. M. Hansson, H. Jung, The status of ccfm unintegrated gluon densities, 23-27 April 2003. DIS 2003, St. Petersburg, Russia. arXiv:hep-ph/0309009

16. G. Marchesini, B. Webber, Nucl. Phys. B 349, 617 (1991)

17. G. Marchesini, B. Webber, Nucl. Phys. B 386, 215 (1992)

18. S. Aid, et al. (H1 Collaboration), Nucl. Phys. B 470, 3 (1996). 96-039, arXiv:hep-ex/9603004

19. C. Adloff, et al. (H1 Collaboration), Eur. Phys. J. C 21, 33 (2001). DESY 00-181, arXiv:hep-ex/0012053

20. M. Derrick et al. (ZEUS Collaboration), Z. Phys. C 72, 399 (1996). DESY 96-076, arXiv:hep-ex/9607002

21. S. Chekanov et al. (ZEUS Collaboration), Eur. Phys. J. C 21, 443 (2001). DESY 01-064, arXiv:hep-ex/0105090

22. H.L. Lai et al. (CTEQ Collaboration), Eur. Phys. J. C 12, 375 (2000). arXiv:hep-ph/9903282

23. M. Glück, E. Reya, A. Vogt, Z. Phys. C 67, 433 (1995)

24. J. Blümlein, On the $k_{t}$ dependent gluon density of the proton, in Proc. of the Workshop on Deep Inelastic Scattering and $Q C D$, ed. by J. Laporte, Y. Sirois (1995). DESY 95-121 and arXiv:hep-ph/9506403

25. S. Baranov, N. Zotov, Phys. Lett. B 458, 389 (1999)

26. S. Baranov, N. Zotov, Phys. Lett. B 491, 111 (2000)

27. J. Kwiecinski, A. Martin, A. Stasto, Phys. Rev. D 56, 3991 (1997)

28. K. Golec-Biernat, M. Wusthoff, Phys. Rev. D 60, 114023 (1999). arXiv:hep-ph/9903358
29. M.A. Kimber, A.D. Martin, M.G. Ryskin, Phys. Rev. D 63, 114027 (2001). arXiv:hep-ph/0101348

30. V. Saleev, N. Zotov, Mod. Phys. Lett. A 9, 151 (1994)

31. A.V. Lipatov, N.P. Zotov, Eur. Phys. J. C 27, 87 (2003). arXiv:hep-ph/0210310

32. S.P. Baranov, N.P. Zotov, J. Phys. G 29, 1395 (2003). arXiv:hep-ph/0302022

33. S.P. Baranov, Phys. Rev. D 66, 114003 (2002)

34. F. Hautmann, Phys. Lett. B 535, 159 (2002). arXiv:hep-ph/ 0203140

35. S.P. Baranov, A.V. Lipatov, N.P. Zotov, Phys. Rev. D 78, 014025 (2008). arXiv:0805.4821v2 [hep-ph]

36. M. Deak, F. Schwennsen, J. High Energy Phys. 09, 035 (2008). arXiv:0805.3763v2 [hep-ph]

37. S. Marzani, R.D. Ball, Nucl. Phys. B 814, 246 (2009). arXiv:0812.3602v2 [hep-ph]

38. M. Deak, F. Hautmann, H. Jung, K. Kutak, J. High Energy Phys. 09, 121 (2009). arXiv:0908.0538v1 [hep-ph]

39. E. Boos et al., Generic user process interface for event generators (2001). arXiv:hep-ph/0109068

40. G. Ingelman, A. Edin, J. Rathsman, Comput. Phys. Commun. 101, 108 (1997). arXiv:hep-ph/9605286

41. S. Kawabata, Comput. Phys. Commun. 88, 309 (1995)

42. E.J. Eichten, C. Quigg, Phys. Rev. D 52, 1726-1728 (1995) 